\title{
Rapid ascending aorta stiffening in bicuspid aortic valve on serial cardiovascular magnetic resonance evaluation: comparison with connective tissue disorders
}

\author{
Alejandro Perez-Casares ${ }^{1}$, Audrey Dionne ${ }^{1,2}$, Kimberlee Gauvreau ${ }^{1,2}$ and Ashwin Prakash ${ }^{1,2^{*}}$
}

\begin{abstract}
Background: Aortic stiffness has been shown to be abnormal in patients with bicuspid aortic valve (BAV), and is considered a component of the aortopathy associated with this condition. Progressive aortic stiffening associated with aging has been previously described in normal adults. However, it is not known if aging related aortic stiffening occurs at the same rate in BAV patients. We determined the longitudinal rate of decline in segmental distensibility in BAV patients using serial cardiovascular magnetic resonance (CMR) studies, and compared to previously published results from a group of patients with connective tissue disorders (CTD).

Methods: A retrospective review of CMR and clinical data on children and adults with BAV ( $n=49,73 \%$ male; $23 \pm 11$ years) with at least two CMRs (total 98 examinations) over a median follow-up of 4.1 years (range 1-9 years) was performed to measure aortic distensibility at the ascending (AAO) and descending aorta (DAo). Longitudinal changes in aortic stiffness were assessed using linear mixed-effects modeling. The comparison group of CTD patients had a similar age and gender profile ( $n=50,64 \%$ male; $20.6 \pm 12$ years).

Results: Compared to CTD patients, BAV patients had a more distensible AAo early in life but showed a steeper decline in distensibility on serial examinations [mean 10-year decline in AAo distensibility $\left(\times 10^{-3} \mathrm{mmHg}^{-1}\right) 2.4$ in BAV vs 1.3 in CTD, $p=0.005]$. In contrast, the DAo was more distensible in BAV patients throughout the age spectrum, and DAo distensibility declined with aging at a rate similar to CTD patients [mean 10 year decline in DAo distensibility $\left(\times 10^{-3} \mathrm{mmHg}^{-1}\right) 0.3$ in BAV vs 0.4 in CTD, $\left.p=0.58\right]$.

Conclusions: On serial CMR measurements, AAo distensibility declined at significantly steeper rate in BAV patients compared to a comparison group with CTDs, while DAo distensibility declined at similar rates in both groups. These findings offer new mechanistic insights into the differing pathogenesis of the aortopathy seen in BAV and CTD patients.
\end{abstract}

Keywords: Bicuspid aortic valve, Connective tissue disorder, Aortic stiffness, Cardiovascular magnetic resonance

\section{Background}

Bicuspid aortic valve (BAV) is the most common congenital heart defect, with a prevalence of $1-2 \%$ in the

\footnotetext{
*Correspondence: ashwin.prakash@cardio.chboston.org

1 Department of Cardiology, Boston Children's Hospital, Boston, MA, USA

Full list of author information is available at the end of the article
}

general population [1]. Many (40-80\%) of these patients will develop ascending aortic (AAo) dilation $[2,3]$. There is an ongoing debate whether the etiology of this dilatation is due to intrinsic aortic wall abnormalities or abnormal flow dynamics related to valve abnormality, or both $[4,5]$. Although often benign, the aortopathy in BAV is progressive and can lead to dissection and death $[3,6]$. Increased 
aortic stiffness has been reported in BAV patients by several investigators and this is considered to be an important component of the aortopathy associated with BAV $[7,8]$. Progressive aortic stiffening is associated with aging in normal adults, and in patients with a connective tissue disorder (CTD) [9]. However, it is not known if aging related aortic stiffening is accelerated in BAV patients. In order to improve our mechanistic understanding of the aortopathy associated with BAV, we determined the longitudinal rate of change in segmental aortic stiffness in BAV patients using serial cardiovascular magnetic resonance (CMR), and compared it with patients with CTDs.

\section{Methods}

A retrospective study of existing clinical data at Boston Children's Hospital from January 2005 through March 2019 was performed. The Department of Cardiology's Scientific Review Committee and the Boston Children's Hospital's Committee on Clinical Investigation gave permission for a retrospective chart review.

\section{Subjects}

Children and adults with BAV with least 2 available clinically indicated CMR examinations (minimum interval in the supine position using commercial oscillometric blood pressure recorders. Electrocardiogram (ECG)gated 2 dimensional cine balanced steady-state free precession (bSSFP) imaging of the left ventricular outflow tract in 2 orthogonal planes was performed that were then used to plan a stack of cine bSSFP images in the short axis of the AAo and the descending aorta (DAo) as previously described [11]. Sievers classification was used to describe the type of BAV [12]. Cine bSSFP images were analyzed at 2 locations (AAo and thoracic DAo) to calculate parameters of stiffness as previously reported [11]. At each location, cross-sectional area was measured by a single observer using manual planimetry at both peak systole and end-diastole. Images were cross-referenced with 2 long axis planes to select the appropriate short-axis slice perpendicular to the aorta. Aortic stiffness was assessed using the following parameters as previously described [11]:

$$
\text { Strain }=\frac{\text { Systolic area }- \text { Diastolic area }}{\text { Diastolic area }}
$$

$$
\text { Distensibility }(A D)=\frac{\text { Strain }}{\text { Brachial pulse pressure }}
$$

$$
\beta \text { stiffness index }=\frac{\ln (\text { systolic blood pressure/diastolic blood pressure })}{\text { Strain }}
$$

between examinations 1 year) were included. Patients with associated complex congenital heart disease (including coarctation of the aorta), a significant shunt lesion, a genetic syndrome, or a CTD were excluded. Patients with unicuspid aortic valve are considered a morphological subset of patients with BAV, sharing complications such us valvar dysfunction and AAo dilation, and were therefore included. We compared the longitudinal changes in aortic stiffness in BAV patients to a group of patients with CTD. Longitudinal changes in aortic stiffness in this CTD group were analyzed using identical techniques and have been previously published by our laboratory [10]. Demographic, clinical and surgical data were abstracted from the hospital's electronic medical record.

\section{CMR imaging and analysis}

CMR was performed for clinical indication using a commercially available whole-body scanner (Achieva; Philips Healthcare, Best, The Netherlands). In young patients who could not cooperate with breathing instructions, the examination was performed under general anesthesia. Brachial artery blood pressure was measured in the right arm before each examination
Measurements were performed using commercially available software $\left(\mathrm{cvi}^{42}\right.$ version 5.10 , Circle Cardiovascular Imaging Inc. Calgary, Alberta, Canada). Height and weight were measured at each CMR examination and body surface area (BSA) was calculated using the Haycock formula [13]. The rate of AAo dilation was calculated as the change in the BSA-adjusted systolic cross-sectional area between the 2 CMR examinations. The severity of aortic regurgitation was categorized semi-quantitatively on phase-contrast CMR flow measurements, whenever possible. The severity of aortic stenosis was categorized semi-quantitatively on echocardiography performed within 1 year of the CMR examination, when available.

\section{Statistical analysis}

The longitudinal change in aortic stiffness parameters was evaluated through linear mixed-effects modeling and expressed as mean change over 10 years with $95 \%$ confidence intervals. Differences in slopes between males and females and other patient subgroups were evaluated using interaction terms. Spearman correlation coefficients were used to assess associations between change in 
stiffness parameter over time and size of the AAo. Baseline patient characteristics were compared between BAV and CTD patients using Fisher's exact test and the twosample t test. For all analyses, a p value less than 0.05 was considered to be statistically significant. Statistical analysis was performed using Stata (version 13.0; Stata Corporation, College Station, Texas, USA).

Table 1 Patient characteristics

\begin{tabular}{|c|c|c|c|}
\hline Parameter & BAV $(n=49)$ & CTD $(n=50)$ & $p$ value \\
\hline Age at first CMR (years) & $23.0 \pm 11$ & $20.6 \pm 12$ & 0.30 \\
\hline Male gender & $36(73 \%)$ & $32(64 \%)$ & 0.26 \\
\hline Type of BAV & & $\mathrm{N} / \mathrm{A}$ & $\mathrm{N} / \mathrm{A}$ \\
\hline Left-right & $22(45 \%)$ & & \\
\hline Right-non & $11(22 \%)$ & & \\
\hline Left-non & $1(2 \%)$ & & \\
\hline Unicuspid & 15 (31\%) & & \\
\hline Type of CTD & N/A & & $\mathrm{N} / \mathrm{A}$ \\
\hline Marfan syndrome & & $26(52 \%)$ & \\
\hline Nonspecific CTD & & $14(28 \%)$ & \\
\hline Loeys-Dietz syndrome & & $7(14 \%)$ & \\
\hline Ehlers-Danlos syndrome & & $3(6 \%)$ & \\
\hline Aortic regurgitation & & & $<0.001$ \\
\hline None/trivial & $12(25 \%)$ & 47 (94\%) & \\
\hline Mild & $30(63 \%)$ & $3(6 \%)$ & \\
\hline Moderate & $5(10 \%)$ & 0 & \\
\hline Severe & $1(2 \%)$ & 0 & \\
\hline Not available & $1(2 \%)$ & $0(0 \%)$ & \\
\hline Aortic stenosis & & & $<0.001$ \\
\hline Mild or less & $23(50 \%)$ & $50(100 \%)$ & \\
\hline Moderate & $22(48 \%)$ & 0 & \\
\hline Severe & $1(2 \%)$ & 0 & \\
\hline Not available & $2(4 \%)$ & 0 & \\
\hline$\beta$ blocker and/or ARB use & $30(61 \%)$ & $24(48 \%)$ & 0.22 \\
\hline
\end{tabular}

$A R B$ denotes angiotensin receptor blocker, $B A V$ bicuspid aortic valve, CTD connective tissue disorders. The severity of aortic regurgitation and stenosis was assessed by echocardiography

\section{Results}

\section{Subjects}

Characteristics of BAV subjects and the comparison group of CTD patients are summarized in Table 1. The study population with BAV consisted predominantly of young and middle-age adults with a median age of 18 years, but $14 / 49(29 \%)$ were children $<18$ years of age. A total of 98 CMR examinations were analyzed in 49 patients ( 2 examinations per patient, median duration between examinations 4.1 years, range 1.0-9.4 years). Consistent with prior studies in patients with BAV, there was male preponderance (73\%). Valve stenosis or regurgitation was common, but mild or moderate in severity in a majority of patients. A minority of patients were receiving a $\beta$-blocker or an angiotensin converting enzyme inhibitor. The results of the comparison group of CTD patients have been previously reported. The median duration between examinations in the CTD group was 3.9 years (range 1.0 to 13.2). The BAV and CTD groups were similar in their age and gender distribution. The BAV group patients had higher prevalence of aortic stenosis or regurgitation. Rates of $\beta$-blocker and angiotensin receptor blocker use were similar among groups.

\section{Longitudinal change in aortic stiffness in BAV}

The results of linear mixed-effects modeling to determine the longitudinal rate of change in stiffness parameters are summarized in Table 2 and Fig. 1. Patient-level longitudinal changes are depicted in Fig. 2. Both the AAo and DAo became stiffer with aging, with progressive decline in aortic strain and distensibility, and increase in the $\beta$-stiffness index. However, the rate aortic stiffening was steeper at the AAo. We also explored non-linear models and found that they did not provide an improved fit compared to the linear models.

For each stiffness parameter, the rate of longitudinal change did not vary significantly by baseline severity of aortic stenosis or regurgitation, or by type of BAV. There were no gender differences in the rate of longitudinal

Table 2 Comparison of serial change in aortic stiffness in BAV and CTD

\begin{tabular}{lccc}
\hline Parameter & BAV Mean $\mathbf{1 0}$ year change $(\mathbf{n}=\mathbf{4 9})$ & CTD Mean $\mathbf{1 0}$ year change $(\mathbf{n}=\mathbf{5 0})^{*}$ & $\boldsymbol{p}$ value \\
\hline Ascending aorta (AAO) & $-10.3(-13.7,-6.9)$ & $-3.7(-5.3,-2.0)$ & $<0.001$ \\
Strain (\%) & $-2.4(-3.2,-1.7)$ & $-1.3(-1.8,-0.9)$ & 0.005 \\
Distensibility $\left(\times 10^{-3} \mathrm{mmHg}^{-1}\right)$ & $1.5(1.0,2.0)$ & $0.6(0.1,0.2)$ & $<0.001$ \\
$\beta-$ stiffness index & & $-2.5(-3.7,-1.3)$ & 0.28 \\
Descending thoracic aorta (DAo) & $-3.7(-5.8,-1.5)$ & $-1.0(-1.3,-0.6)$ & 0.37 \\
Strain (\%) & $-1.2(-1.8,-0.7)$ & $0.4(0.2,0.6)$ & 0.58 \\
Distensibility $\left(\times 10^{-3} \mathrm{mmHg}^{-1}\right)$ & $0.3(0.1,0.6)$ & \\
$\beta$-stiffness index &
\end{tabular}

*Data on CTD patients is derived from previously published data from our laboratory [10] 

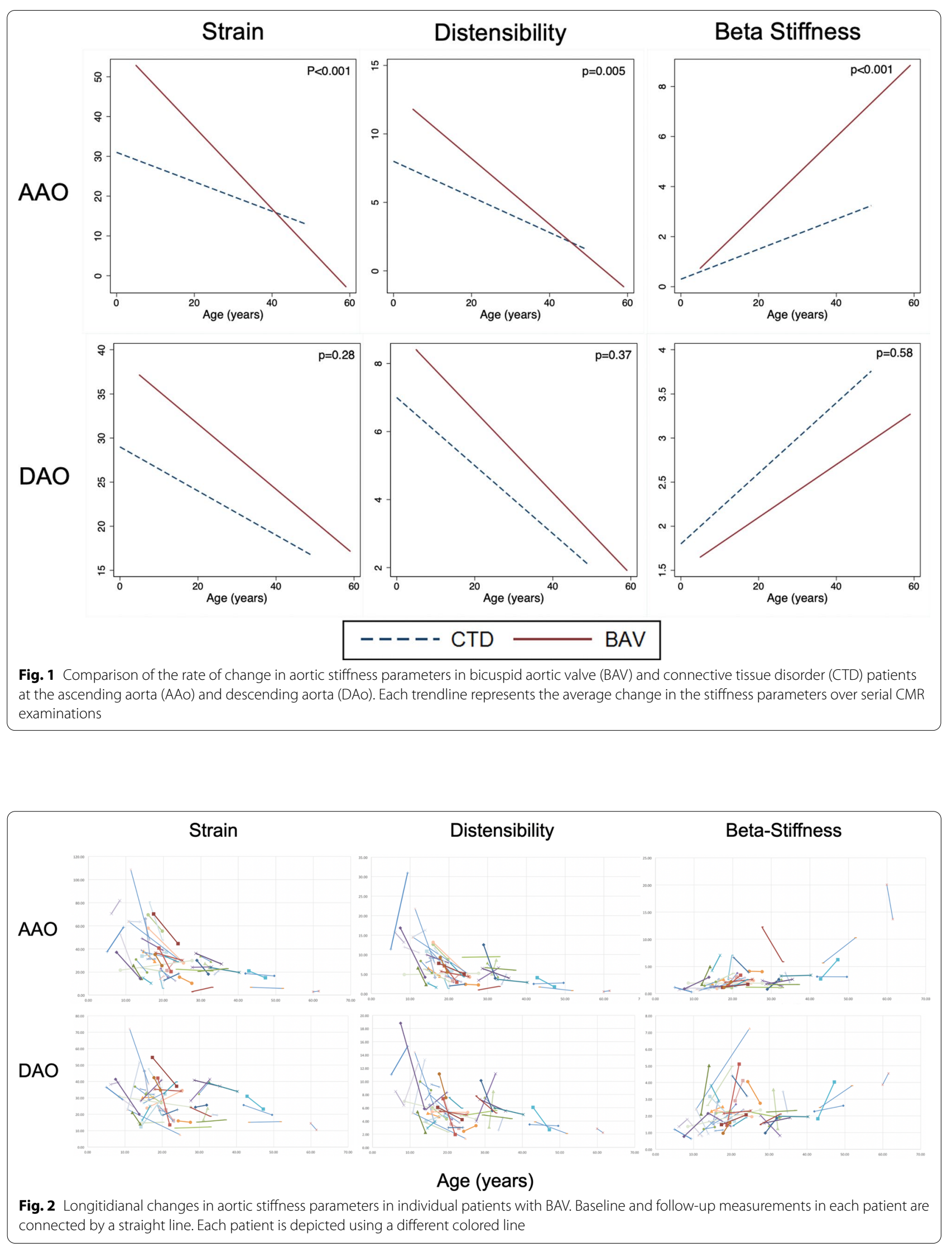
change in strain or distensibility at the AAo or DAo. However, the rate of increase in the $\beta$-stiffness index at the AAo was faster in females (mean 10-year change 2.4 vs 1.0 in males).

\section{Comparison with CTD}

Detailed results from the CTD group have been previously published [10]. Comparisons between the BAV and CTD groups are presented in Table 2 and Fig. 1. As expected, there was progressive aortic stiffening with increasing age at both the AAo and DAo. However, as seen in Fig. 1, the BAV patients started out with a more distensible AAo earlier in life, but showed a steeper rate of AAo stiffening compared to the CTD patients, resulting in a stiffer AAo at older ages. As seen in Table 2, the mean 10-year rate of change in all the stiffness parameters at the AAo was statistically different between the two groups. In contrast, at the DAo, the BAV patients continued to show a more distensible DAo compared to the CTD group throughout the studied age spectrum and there was no statistically significant difference in the rate of change in stiffness parameters.

\section{Discussion}

In serial CMR analyses of children and young adults with BAV and CTD, aortic strain and distensibility progressively decreased with age. Compared to the CTD group, BAV patients had more distensible AAo early in life but showed a steeper decline in distensibility on serial examinations. In contrast, the DAo was more distensible in BAV patients throughout the age spectrum, and DAo distensibility declined with aging at a rate similar to CTD patients.

\section{Longitudinal change in aortic stiffness in BAV and comparison with CTD}

It has been previously reported that patients with BAV have a stiffer aorta [7, 8]. It is also known that aortic stiffness increases with aging [14, 15]. However, the longitudinal rate of change in aortic stiffness in BAV has not been previously studied. Our results show that aortic stiffness in BAV patients increases at both the AAo and DAo, but the rate of increase is steeper at the AAo, which is the segment often associated with dilation and histopathological wall abnormalities. On comparison with a cohort of patients with CTD-associated aortopathy from our own laboratory, the rate of AAo stiffening was significantly steeper in BAV patients [10]. In contrast, at the DAo, the BAV patients had a more distensible aorta at all ages with a similar age related decline in distensibility, compared to CTD. Notably, although the BAV group had a higher prevalence of aortic valve stenosis and regurgitation, this did not significantly impact the rate of longitudinal change in stiffness parameters.
The rate of aortic stiffening in CTD patients has been previously shown by us to be similar to normal adults [9]. We decided to compare the rate of stiffening in BAV with the previously reported cohort of CTD patients, because these two disorders represent two common etiologies for aortic dilation in children and young adults. The mechanism underlying a faster rate of stiffening of the AAo in BAV are unclear. Both BAV and CTD are associated with histopathologic aortic wall abnormalities that result in stiffening, but there are key differences in their pathogenetic mechanisms. In CTDs, the underlying genetic abnormality results in alterations in the composition of the aortic wall. In BAV, although histologic abnormalities of the aortic wall are noted, an additional role for hemodynamic factors related to increased wall shear stress and abnormal flow profile through the valve has been previously suggested $[4,5,16,17]$. In this light, it is interesting to note that in this cohort, the baseline distensibility was nearly normal in BAV patients, while CTD had significantly stiff AAos even at baseline. Although this requires further investigation, it could be speculated that increased hemodynamic stress on the AAo wall contributes to the steeper stiffening of the AAo in the BAV patients. These hemodynamic factors are not present in the DAo, where the rate of aortic stiffening is similar in CTD and BAV patients.

\section{Comparison with other techniques to assess aortic stiffnes and flow}

Other techniques for measuring aortic stiffness such as Displacement Encoding with Stimulated Echoes (DENSE) can be used to measure aortic stiffness [18]. This technique allows assessment of regional variations in aortic strain. However, this technique requires a specialized pulse sequence which is not in routine clinical use and was therefore not available in our patients. 4D flow imaging can provide unique insights into the abnormal flow patterns and wall shear stress in patients with BAV $[16,17]$. However, these assessments also require the use of a specialized pulse sequence that is not in routine clinical use and was therefore not available for this retrospective analysis.

\section{Limitations}

Several limitations of this work are worth considering. First, it suffers from known limitations of a retrospective design, but these are likely offset at least in part by the uniformity of the imaging protocol. Second, there is potential for referral bias, as CMR was not performed uniformly on all patients with BAV seen at our institution, and this may limit generalizability. Third, a retrospective cohort of healthy children and adults was not 
available for direct comparison. However, as noted previously, rates of aortic stiffening in healthy adults have been previously reported, and found by us to be similar to patients with CTD $[9,10]$. Finally, a significant proportion of patients had aortic valve dysfunction (stenosis or regurgitation) and this could potentially impact measurements of stiffness. However, it is reassuring that in multivariable modeling, the rate of change of stiffness was not impacted by presence and severity of valve dysfunction.

\section{Conclusions}

In longitudinal CMR analyses, children and young adults with BAV had a more distensible AAo compared to CTD patients early in life but showed a steeper decline in distensibility on serial examinations. In contrast, the DAo was more distensible in BAV patients throughout the age spectrum, with a rate of decline similar to CTD patients. These results offer mechanistic insights into the varying pathogenesis of aortopathy in BAV and CTD patients.

\begin{abstract}
Abbreviations
AAo: Ascending aorta; BAV: Bicuspid aortic valve; BSA: Body surface area; bSSFP: Balanced steady state free precession; CMR: Cardiovascular magnetic resonance; CTD: Connective tissue disorder; DAO: Descending thoracic aorta; ECG: Electrocardiogram.
\end{abstract}

\section{Acknowledgements}

None

\section{Authors' contributions}

AP-C contributed with study conception and design, data acquisition, and drafting the manuscript; AD contributed with data acquisition and manuscript revision; KG performed statistical analyses; AP contributed with study conception and design, data analysis, manuscript revision and overall supervision of the project. All authors read and approved the final manuscript.

\section{Funding}

Funding provided by Daniel Bravo Foundation, The Spanish Society for congenital heart disease research and Hospital Sant Joan de Deu (Esplugues de Llobregat, Barcelona, Spain).

\section{Availability of data and materials}

The datasets used and/or analyzed during the current study are available from the corresponding author on reasonable request.

\section{Ethics approval and consent to participate}

The Department of Cardiology's Scientific Review Committee and the Boston Children's Hospital's Committee on Clinical Investigation gave permission for a retrospective chart review.

\section{Consent for publication}

Not applicable.

\section{Competing interests}

The authors declare that they have no competing interests.

\section{Author details}

${ }^{1}$ Department of Cardiology, Boston Children's Hospital, Boston, MA, USA.

2 Department of Pediatrics, Harvard Medical School, 300 Longwood Avenue, Boston, MA 02115, USA.

Received: 3 July 2020 Accepted: 26 January 2021

Published online: 22 February 2021
References

1. Ward C. Clinical significance of the bicuspid aortic valve. Heart. 2000:83:81-5.

2. Nistri S, Sorbo MD, Marin M, Palisi M, Scognamiglio R, Thiene G. Aortic root dilatation in young men with normally functioning bicuspid aortic valves. Heart. 1999;82:19-22.

3. Della Corte A, Bancone C, Quarto C, Dialetto G, Covino FE, Scardone M, Caianiello G, Cotrufo M. Predictors of ascending aortic dilatation with bicuspid aortic valve: a wide spectrum of disease expression. Eur J Cardiothorac Surg. 2007;31:397-404.

4. Sievers HH, Sievers HL. Aortopathy in bicuspid aortic valve disease genes or hemodynamics? or Scylla and Charybdis? Eur J Cardiothorac Surg. 2011;39:803-4.

5. Girdauskas E, Borger MA, Secknus MA, Girdauskas G, Kuntze T. Is aortopathy in bicuspid aortic valve disease a congenital defect or a result of abnormal hemodynamics? A critical reappraisal of a one-sided argument. Eur J Cardiothorac Surg. 2011;39:809-14.

6. Wojnarski CM, Svensson LG, Roselli EE, Idrees JJ, Lowry AM, Ehrlinger J, Pettersson GB, Gillinov AM, Johnston DR, Soltesz EG, Navia JL, Hammer DF, Griffin B, Thamilarasan M, Kalahasti V, Sabik JF 3rd, Blackstone EH, Lytle BW. Aortic dissection in patients with bicuspid aortic valve-associated aneurysms. Ann Thorac Surg. 2015;100:1666-73.

7. de Wit A, Vis K, Jeremy RW. Aortic stiffness in heritable aortopathies: relationship to aneurysm growth rate. Heart Lung Circ. 2013;22:3-11.

8. Boonyasirinant T, Rajiah P, Flamm SD. Abnormal aortic stiffness in patients with bicuspid aortic valve: phenotypic variation determined by magnetic resonance imaging. Int J Cardiovasc Imaging. 2019;35:133-41.

9. Kim EK, Chang SA, Jang SY, Kim Y, Kim SM, Oh JK, Choe YH, Kim DK. Assessment of regional aortic stiffness with cardiac magnetic resonance imaging in a healthy Asian population. Int J Cardiovasc Imaging. 2013:29(Suppl 1):57-64.

10. Merlocco A, Lacro RV, Gauvreau K, Rabideau N, Singh MN, Prakash A. Longitudinal changes in segmental aortic stiffness determined by cardiac magnetic resonance in children and young adults with connective tissue disorders (the Marfan, Loeys-Dietz, and Ehlers-Danlos Syndromes, and Nonspecific Connective Tissue Disorders). Am J Cardiol. 2017;120:1214-9.

11. Prakash A, Adlakha H, Rabideau N, Hass CJ, Morris SA, Geva T, Gauvreau K, Singh MN, Lacro RV. Segmental aortic stiffness in children and young adults with connective tissue disorders: relationships with age, aortic size, rate of dilation, and surgical root replacement. Circulation. 2015;132:595-602.

12. Sievers HH, Schmidtke C. A classification system for the bicuspid aortic valve from 304 surgical specimens. J Thorac Cardiovasc Surg. 2007;133:1226-33.

13. Haycock GB, Schwartz GJ, Wisotsky DH. Geometric method for measuring body surface area: a height-weight formula validated in infants, children, and adults. J Pediatr. 1978;93:62-6.

14. Redheuil A, Yu WC, Wu CO, Mousseaux E, de Cesare A, Yan R, Kachenoura N, Bluemke D, Lima JA. Reduced ascending aortic strain and distensibility: earliest manifestations of vascular aging in humans. Hypertension. 2010;55:319-26.

15. Kim YG, Sun BJ, Park GM, Han S, Kim DH, Song JM, Kang DH, Song JK. Aortopathy and bicuspid aortic valve: haemodynamic burden is main contributor to aortic dilatation. Heart. 2012;98:1822-7.

16. Barker AJ, Markl M. The role of hemodynamics in bicuspid aortic valve disease. Eur J Cardiothorac Surg. 2011;39:805-6.

17. Barker AJ, Markl M, Burk J, Lorenz R, Bock J, Bauer S, Schulz-Menger J, von Knobelsdorff-Brenkenhoff F. Bicuspid aortic valve is associated with altered wall shear stress in the ascending aorta. Circ Cardiovasc Imaging. 2012;5:457-66

18. Wilson JS, Taylor WR, Oshinski J. Assessment of the regional distribution of normalized circumferential strain in the thoracic and abdominal aorta using DENSE cardiovascular magnetic resonance. J Cardiovasc Magn Reson. 2019;21:59.

\section{Publisher's Note}

Springer Nature remains neutral with regard to jurisdictional claims in published maps and institutional affiliations. 